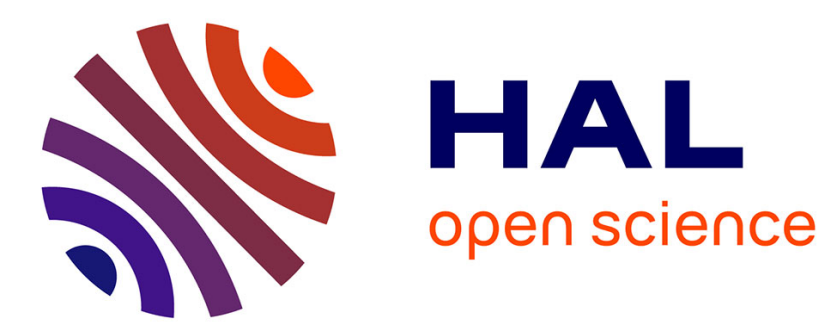

\title{
Prospections écologiques et sylvicoles dans les cédraies du Belezma et de l'Aurès à la recherche de peuplements semenciers et d'arbres plus.
}

Abdelkader Harfouche, Abdallah Nedjahi

\section{- To cite this version:}

Abdelkader Harfouche, Abdallah Nedjahi. Prospections écologiques et sylvicoles dans les cédraies du Belezma et de l'Aurès à la recherche de peuplements semenciers et d'arbres plus.. Revue forestière française, 2003, 55 (2), pp.113-122. 10.4267/2042/5162 . hal-03449359

\author{
HAL Id: hal-03449359 \\ https://hal.science/hal-03449359
}

Submitted on 25 Nov 2021

HAL is a multi-disciplinary open access archive for the deposit and dissemination of scientific research documents, whether they are published or not. The documents may come from teaching and research institutions in France or abroad, or from public or private research centers.
L'archive ouverte pluridisciplinaire HAL, est destinée au dépôt et à la diffusion de documents scientifiques de niveau recherche, publiés ou non, émanant des établissements d'enseignement et de recherche français ou étrangers, des laboratoires publics ou privés. 


\title{
BIOLOGIE ET ECOLOGIE
}

\section{Prospections ÉcologiQUes et SyLVICOLES DANS LES CÉDRAIES DU BÉLÉZMA ET DE L'AURÈS À LA RECHERCHE DE PEUPLEMENTS SEMENCIERS ET D’ARBRES PLUS}

\author{
Abdelkader Harfouche - Abdellah Nedjahı
}

Le Cèdre de l'Atlas (Cedrus atlantica Manetti) est, en Algérie, un arbre de première grandeur des forêts de montagne où il occupe une surface d'environ 30000 ha dans les Atlas tellien et saharien. Il apparaît à partir de $1300 \mathrm{~m}$ d'altitude en versant nord et vers $1400 \mathrm{~m}$ en versant sud ; mais il peut descendre plus bas (jusqu'à 900-1 $000 \mathrm{~m}$ ) le long des ravins où existent des conditions pédo-microclimatiques propices. Des plantations effectuées avec succès dans l'Atlas tellien et l'Aurès témoignent de l'aptitude de cette espèce à coloniser des espaces et milieux nouveaux et, à ce titre, le Cèdre se présente comme une essence de reboisement majeure dans l'étage montagnard, au-dessus de 1000 à $1200 \mathrm{~m}$ d'altitude selon l'exposition. En Algérie, l'aire actuelle du Cèdre de l'Atlas est très morcelée (figure 1, p. 115) ; ce morcellement s'explique par les grands changements climatiques survenus durant le Quaternaire récent qui ont vu le recul de la cédraie et l'apparition d'espèces concurrentes plus adaptées aux climats modernes. La configuration de cette aire, caractérisée par une diversité écologique notable et un isolement plus ou moins grand des populations, dont certaines de faible taille, a induit une différenciation perceptible de l'espèce. L'existence d'écotypes différents par rapport aux facteurs climatiques (stations allant du semi-aride supérieur à l'humide) et édaphiques (variété de substrats aussi bien siliceux que calcaires) ainsi que de morphotypes divers (formes glauca et viridis, forme à port pleureur, etc.) sont des témoins facilement observables d'une différenciation géographique et individuelle d'origine probablement génétique.

Des recherches récentes ont révélé une grande variabilité géographique pour des caractères du pollen (Derridj, 1990), des graines (Harfouche, 1992) ou des stomates (Arbez et al., 1978 ; Berka, 1997) ainsi que pour divers caractères d'adaptation, de croissance et de forme (Arbez et al., 1978 ; Pradal, 1979 ; Ferrandès, 1986 ; Bariteau et Ferrandès, 1992). Cette diversité intra-spécifique est un atout pour les programmes de plantation de Cèdre aussi bien pour la reconstitution des peuplements dans son aire naturelle que pour le reboisement de terrains à l'intérieur d'une aire potentielle dont les contours ont été grossièrement définis. En Algérie, l'aire potentielle de l'espèce pourrait s'étendre à tout l'Atlas frais et froid, là où les précipitations et les brouillards printaniers ne sont pas rares (Maire, 1926). Cependant, un programme sérieux de sélection d'écotypes et d'individus résistant à la sécheresse et au calcaire pourrait offrir de plus grandes possibilités de reboisement. En dehors de son aire naturelle, le Cèdre de l'Atlas a été introduit avec succès, dès les années 1860, dans le Sud-Est de la France (Toth, 1972 ; Pradal, 1979) où l'on compterait aujourd'hui quelque 20000 ha. On estime à 200000 ha l'aire potentielle du Cèdre dans la zone supra-méditerranéenne française où la recherche d'écotypes croissant bien sur calcaires reste un objectif de sélection essentiel (Bariteau et Ferrandès, 1992). En Italie, des plan- 
tations de Cèdre de l'Atlas ont été réalisées dès le début du XXe siècle en Toscane, dans le Nord de la péninsule ; on y compte plus de 1000 ha (Nedjahi, 1988). Le Cèdre de l'Atlas a été introduit avec succès dans le Nord-Est des États-Unis (Pennsylvanie, Massachussetts, État de New York) - où il prend une place de plus en plus importante dans les projets - et à grande échelle dans certaines régions de l'ex-URSS (Crimée et Caucase) (Toth, 1980 ; Nedjahi, 1988). Des introductions à titre expérimental ont été effectuées dans de nombreux pays dont la Hongrie, la Yougoslavie, l'Espagne, le Portugal, la Grande Bretagne et la Belgique (Nedjahi, 1988).

En Algérie, le développement des plantations et la conservation de la ressource génétique sont fortement imbriqués. La démarche la plus adéquate consisterait à faire la distinction entre reconstitution des cédraies existantes et reboisement des zones potentielles d'introduction; la première option doit utiliser des sources de graines locales afin de maintenir la spécificité des provenances ; la seconde doit employer la ou les provenances ayant fait preuve de leur supériorité dans les tests.

Après une étude monographique succincte, nous présentons, dans une première partie, un compte-rendu des prospections ${ }^{(1)}$ effectuées à la recherche de peuplements semenciers dans les massifs du Bélézma et de l'Aurès (Chélia et Ouled Yacoub). Dans une deuxième partie, nous exposons brièvement un programme pour la création d'un verger à graines de familles via un test de descendances maternelles dans le massif des Ouled Yacoub (Département de Khenchela). Par ailleurs, des possibilités financières nouvelles nous permettent d'envisager en parallèle la mise en place d'un test de provenances restreint réunissant des provenances locales.

\section{DISTRIBUTION GÉOGRAPHIQUE DU CÈDRE DE L'ATLAS}

Le Cèdre de l'Atlas est une espèce endémique d'Afrique du Nord et caractéristique des forêts des étages montagnards au Maroc ( 16000 ha dans le Rif, 116000 ha dans les moyen et haut Atlas) et en Algérie (quelque 30000 ha dans les Atlas tellien et saharien). Globalement, on distingue, en Algérie, les populations de Cèdre de l'Atlas tellien (conditions mésophiles) et les populations de l'Atlas saharien (conditions relativement xérophiles). Les cédraies de l'Atlas tellien se rencontrent dans le massif de l'Ouarsenis (2 000 ha à Théniet El Had, Boucaïd, etc.), l'Atlas blidéen ( 1000 ha à Chréa), le Djurdjura (2 000 ha à Tala Guilef et Tikjda principalement) ainsi que dans les Babors et Tababors (1 $300 \mathrm{ha}$ ). Dans l'Atlas saharien, on les retrouve principalement dans les massifs du Bélézma et de l'Aurès (17 000 ha) ainsi que dans les monts du Hodna ( 8000 ha à Boutaleb). D'un point de vue bioclimatique, les populations de Cèdre colonisent des milieux allant du semi-aride supérieur froid dans certaines stations de l'Aurès et des monts du Hodna (450 à $500 \mathrm{~mm}$ de pluie/an) au subhumide frais à froid dans l'Aurès, le Hodna, l'Ouarsenis et le versant sud du Djurdjura $(600-800 \mathrm{~mm} / \mathrm{an})$ et à l'humide froid dans les Babors (plus de $2000 \mathrm{~mm} / \mathrm{an}$ ), le versant nord du Djurdjura et l'Atlas blidéen (1 200-2 $000 \mathrm{~mm} / \mathrm{an}$ ). En hiver, les peuplements de Cèdre ont fréquemment à supporter des températures de $-11^{\circ}$ à $-15^{\circ} \mathrm{C}$ dans l'Aurès et les monts du Hodna (Abdessemed, 1981).

Au plan géologique, le Cèdre colonise des substrats très variés, schistes néocomiens, grès blancs, grès et quartzites, calcaires massifs, calcaires dolomitiques et dolomie, marno-calcaires et marnes ; sa croissance reste, toutefois, moindre sur les terrains calcaires que sur les roches mères siliceuses, d'autant plus que les sols y sont souvent plus superficiels.

(1) Plusieurs vagues de prospections ont été effectuées en 1989, 1990 et 1991, la dernière conjointement avec l'INRA (représenté par M. Bariteau d'Avignon puis M. Vernier de Nancy). Ces prospections ont été mises à jour et complétées en 1999 et 2000. 
Dans la région de Khenchela (Sud Constantinois), où l'essentiel de nos travaux est concentré, le Cèdre de l'Atlas occupe une superficie d'environ 6000 ha, répartis en deux grands massifs d'égale importance : les Ouled Yacoub et Bouhmama (figure 1, ci-dessous, région 1). Dans le massif des Ouled Yacoub, le Cèdre est accompagné du Chêne vert et du Frêne dimorphe qui constituent le sous-étage climacique du Cèdre dans les Aurès. Des individus remarquables, de très bonne conformation et d'une hauteur atteignant aisément les $30-40 \mathrm{~m}$, se rencontrent dans les stations les plus favorables (ravines au sol profond et humifère).

Figure 1

AIRE DE DISTRIBUTION DU CÈDRE DE L'ATLAS

1- Aurès-Bélézma-Monts du Hodna 2- Babors et Tababors 3-Djurdjura 4- Atlas blidéen 5- Ouarsenis

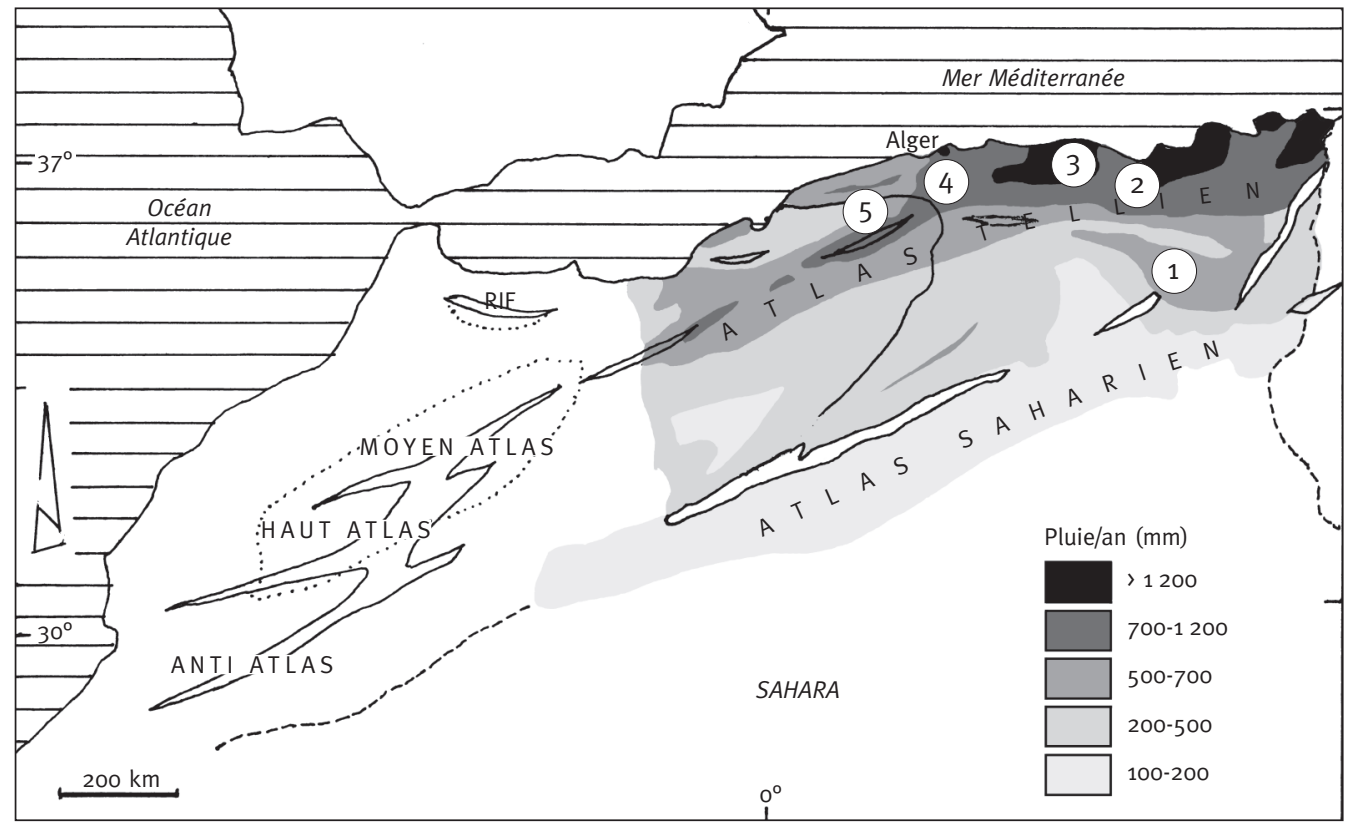

La carte pluviométrique illustre la variété des climats auxquels est soumis le Cèdre de l'Atlas dans son aire en Algérie
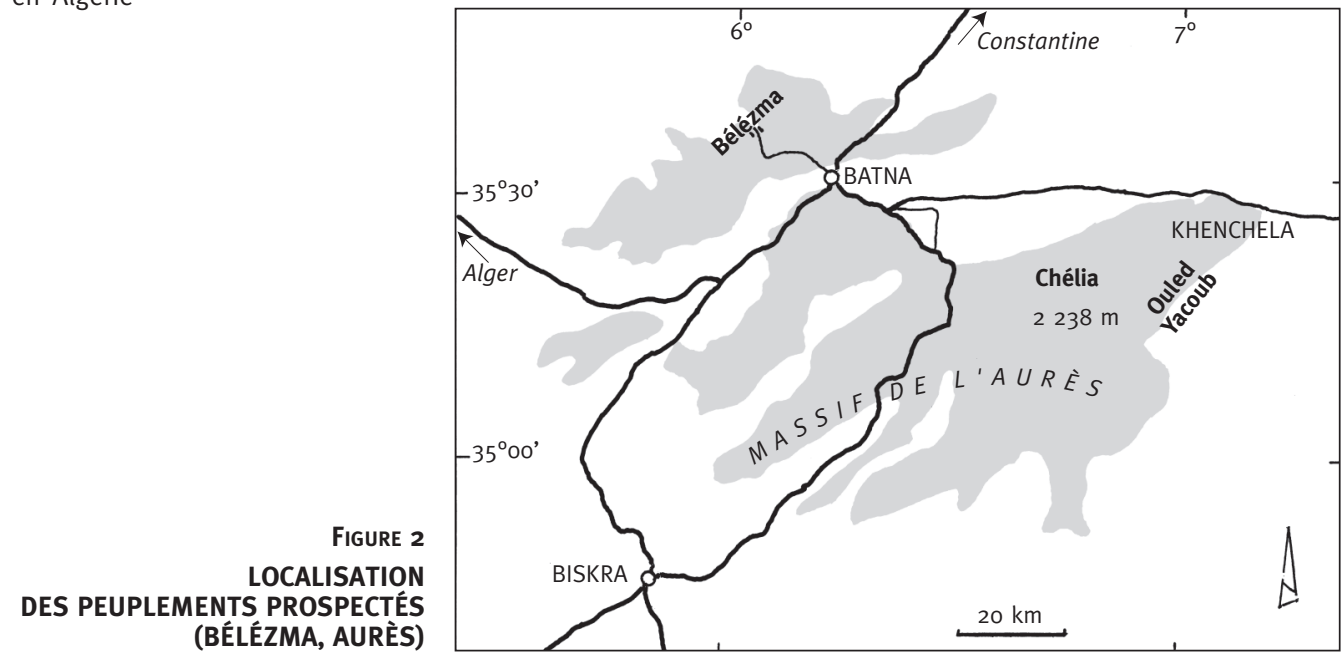


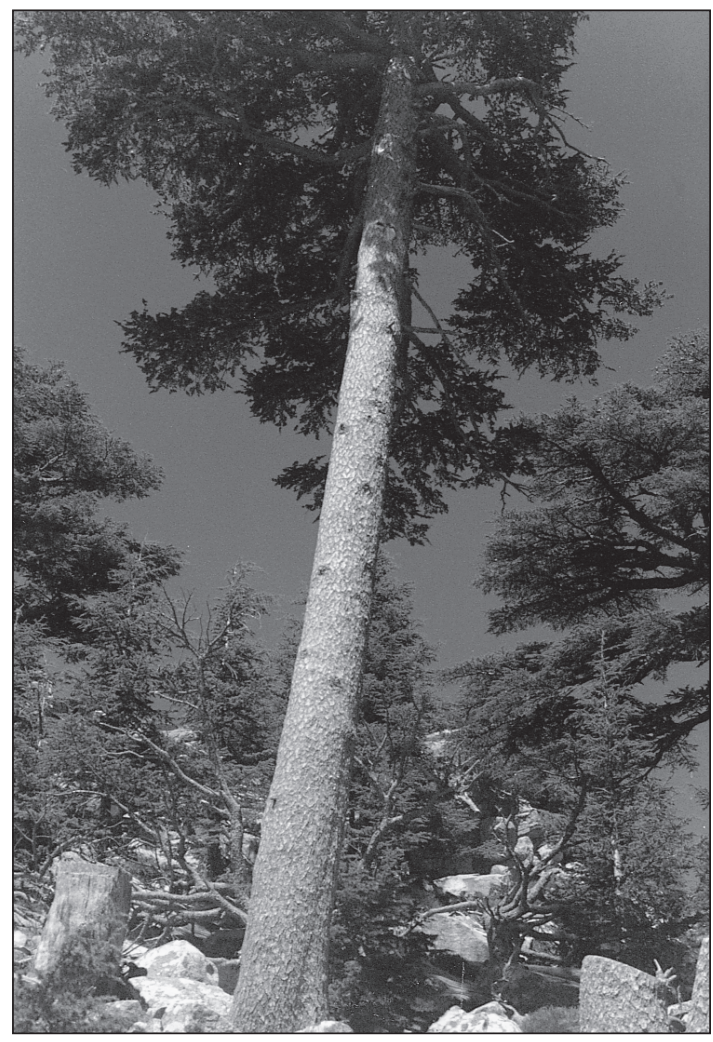

Arbre plus

en forêt des Ouled Yacoub

dans le massif de l'Aurès

Photo A. HARFOUCHE

La régénération s'installe facilement lorsque les conditions d'éclairement et la structure du sol le permettent ; les talus de pistes, bien éclairés et ameublis, sont souvent envahis par des fourrés très vigoureux. La structure des peuplements est, généralement, irrégulière avec une fréquence de bouquets plus ou moins équiennes et de surface variable (de quelques ares à quelques hectares). Une fructification abondante s'observe surtout dans les peuplements clairs et sur les arbres dont la cime est bien dégagée. À l'heure actuelle, la cédraie ne semble pas connaître de problèmes phytosanitaires ; les populations de processionnaires (Thaumetopoea pityocampa et Thaumetopoea bonjeani) sont très discrètes et les cas de maladies cryptogamiques rarissimes. La grande vitalité actuelle du Cèdre dans les Ouled Yacoub laisse supposer que cette espèce est toujours en équilibre avec le milieu. La principale contrainte à la régénération naturelle du massif est le pâturage qui constitue une ressource importante pour les populations riveraines. Tout schéma d'aménagement doit assurer un juste équilibre entre les objectifs de renouvellement des peuplements et la fonction socio-économique de la forêt.

\begin{tabular}{|c|c|c|c|}
\hline Critères & Station 1 & Station 2 & Station 3 \\
\hline Localisation ............. & Versant nord & $\begin{array}{l}\text { Versant nord, haut bassin } \\
\text { de l'oued } \mathrm{El} \mathrm{Ma}\end{array}$ & Col de Telmet \\
\hline Altitude moyenne $\ldots \ldots \ldots$ & $1300 \mathrm{~m}$ & $1500 \mathrm{~m}$ & $1750 \mathrm{~m}$ \\
\hline Substrat géologique $\ldots \ldots \ldots$ & $\begin{array}{l}\text { Calcaire, grès calcaire, } \\
\text { poches de marno-calcaire }\end{array}$ & Calcaire & Calcaire \\
\hline Description $\ldots \ldots \ldots \ldots \ldots$ & $\begin{array}{l}\text { Cédraie mixte à Chêne } \\
\text { vert. } \\
\text { Peuplement vieillissant } \\
\text { sous lequel } \\
\text { la régénération } \\
\text { est presque absente; } \\
\text { meilleure végétation } \\
\text { dans les ravines où } \\
\text { le sol est plus profond }\end{array}$ & $\begin{array}{l}\text { Cédraie pure. } \\
\text { Peuplement âgé, dégradé } \\
\text { où la régénération } \\
\text { est très rare } \\
\text { Affleurements fréquents } \\
\text { des bancs de calcaire }\end{array}$ & $\begin{array}{l}\text { Cédraie pure. } \\
\text { Peuplement âgé } \\
\text { se régénérant mal ; une } \\
\text { étude de souche (fig. } 3 \text {, } \\
\text { ci-contre) révèle un âge } \\
\text { d'environ } 300 \text { ans } \\
\text { (diamètre de } 60 \mathrm{~cm} \text { à } \\
\text { hauteur d'homme) }\end{array}$ \\
\hline
\end{tabular}




\section{PROSPECTIONS}

Elles ont touché les massifs du Bélézma, le Chélia et les Ouled Yacoub dans l'Aurès (figure 1, région 1 ; figure 2 , p. 115). Les principales informations sur les peuplements parcourus sont présentées dans le tableau la (ci-dessous), lb et Ic (pp. 118-119).

\section{Le Bélézma}

Ce massif, d'environ 7000 ha, situé au nord-ouest de la ville de Batna, a un statut de parc national. Il fait partie des étages bioclimatiques semi-aride supérieur et subhumide (surtout), avec une pluviométrie annuelle de 500 à $700 \mathrm{~mm}$. On y observe une grande variété de substrats géologiques dont des grès, des quartzites, des grès calcaires, des calcaires compactes, des calcaires dolomitiques, des marno-calcaires, etc. En versant Nord, la roche compacte est souvent à nu, ne supportant plus que des cèdres adultes et où ne s'observe plus aucune régénération.

\section{Le Chélia (Aurès central)}

Situé au sud-est de la ville de Batna, cette cédraie s'étend sur près de 7000 ha ; c'est ici que culmine le plus haut sommet de l'Aurès à $2328 \mathrm{~m}$ d'altitude. Mais, au-delà de $2200 \mathrm{~m}$, les pelouses prennent le relais de la forêt, bien que des individus isolés s'observent jusqu'à $2300 \mathrm{~m}$ (Abdessemed, 1981). La tranche pluviométrique annuelle, dans ce massif, varie entre 700 et

\section{FIGURE 3}

\section{CROISSANCE ET ACCROISSEMENT EN DIAMÈTRE D'UN ARBRE DE 300 ANS} (Bélézma, Station 3)

On peut constater une chute sensible des accroissements courants annuels au cours des périodes allant de 50 à 300 ans comparativement à celui de la période de o à 50 ans. À la fin de cette période, l'arbre a déjà atteint plus de la moitié de son diamètre final à 300 ans.

\footnotetext{
A : Croissance totale

$B$ : Accroissement moyen annuel

C : Accroissement courant annuel (0-50 ans, 50-100 ans, $100-200$ ans, $200-300$ ans)
}

\begin{tabular}{|c|c|}
\hline Station 4 & Station 5 \\
\hline $\begin{array}{l}\text { Kef Islane, en versant } \\
\text { sud }\end{array}$ & $\begin{array}{l}\text { Flanc nord-nord-est du } \\
\text { Djebel Bordjem }\end{array}$ \\
\hline $1750 \mathrm{~m}$ & $1700 \mathrm{~m}$ \\
\hline Calcaire dolomitique grès & Calcaire, calcaire gréseux \\
\hline $\begin{array}{l}\text { Cédraie pure, arbres } \\
\text { vieillissants ou vieux, } \\
\text { dont certains en mauvais } \\
\text { état sanitaire; } \\
\text { régénération très rare, } \\
\text { station dégradée } \\
\text { (pacage) }\end{array}$ & $\begin{array}{l}\text { Cédraie pure à tendance } \\
\text { mésophile. Peuplement } \\
\text { de très belle venue où } \\
\text { prédominent les arbres } \\
\text { de jeunes futaie. } \\
\text { Phénotypes }{ }^{(1)} \text { (croissance } \\
\text { et forme) de qualité. } \\
\text { À retenir comme } \\
\text { peuplement semencier }\end{array}$ \\
\hline
\end{tabular}

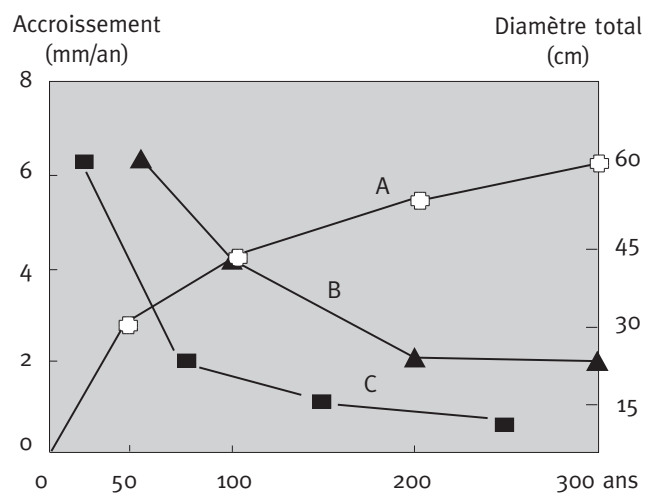

Tableau la Principales caractéristiques des stations du massif de Bélézma

(1) On a mesuré une hauteur de $20 \mathrm{~m}$ et un diamètre (à hauteur d'homme) de $38 \mathrm{~cm}$ sur un arbre de 75 ans, soit un accroissement moyen annuel de plus de $26 \mathrm{~cm}$ en hauteur et d'environ $5 \mathrm{~mm}$ en diamètre. Cette station mérite d'être retenue comme peuplement semencier aussi bien pour les programmes de reconstitution in situ (court terme) que pour le lancement d'un programme de sélection massale en vue de création de vergers à graines de première génération (moyen et long terme). 


\begin{tabular}{|c|c|c|}
\hline Critères & Station 1 & Station 2 \\
\hline Localisation $\ldots \ldots \ldots \ldots \ldots \ldots$ & $\begin{array}{l}\text { Versant sud du Djebel Chélia, } \\
\text { lieu-dit Oued Tidder }\end{array}$ & $\begin{array}{l}\text { Versant sud du Djebel Chélia, } \\
\text { à proximité du chalet ONTF }\end{array}$ \\
\hline Altitude moyenne . . . . . . . . . . . & $1860 \mathrm{~m}$ & $1650 \mathrm{~m}$ \\
\hline Description $\ldots \ldots \ldots \ldots \ldots \ldots$ & $\begin{array}{l}\text { Station relativement mésophile. } \\
\text { Futaie âgée pure; régénération } \\
\text { en périphérie des peuplements } \\
\text { et dans les trouées laissées par } \\
\text { les coupes. Arbres adultes } \\
\text { au phénotype remarquable (dia- } \\
\text { mètre moyen de } 75 \text { à } 80 \mathrm{~cm} \text { et } \\
\text { hauteur moyenne de plus de } \\
30 \text { m). Une étude de souche à } \\
\text { hauteur d'abattage montre des } \\
\text { accroissements notables en } \\
\text { diamètre jusqu'à } 150 \text { ans. À retenir } \\
\text { comme peuplement semencier }\end{array}$ & $\begin{array}{l}\text { Cédraie mixte (Pin d'Alep, Chêne } \\
\text { vert). Mesures sur un sujet de } \\
\text { réserve (âge: } 100 \text { ans; hauteur } \\
\text { totale: } 10 \mathrm{~m} \text {; diamètre à hauteur } \\
\text { d'homme: } 30 \mathrm{~cm}^{(1)} \text { ). Carotte de } \\
\text { sondage montrant des cernes } \\
\text { relativement larges jusqu'à } 50 \text { ans } \\
\text { (diamètre: } 24 \mathrm{~cm} \text {; accroissement } \\
\text { courant annuel: } 4,8 \mathrm{~mm} / \mathrm{an}) \text { puis } \\
\text { cernes de plus en plus fins } \\
\text { jusqu'à } 100 \text { ans (diamètre : } \\
30 \mathrm{~cm} \text {; accroissement courant: } \\
1,2 \mathrm{~mm} / \mathrm{an} \text { ) }\end{array}$ \\
\hline
\end{tabular}

(1) Comparées à la croissance en cédraie d'altitude, ces performances peuvent paraître modestes; cependant, il ne faut pas oublier qu'il s'agit là d'une station qui peut être considérée comme limite pour le Cèdre (il atteint sa limite inférieure vers $1600 \mathrm{~m}$ en versant sud au Chélia). Il est probable que ce type de station représente des écotypes adaptés à des conditions particulières de sécheresse, de température et de sol, et, à ce titre, pourrait se révéler précieux pour le reboisement de zones marginales.

(2) Les arbres, dans cette station, présentent deux phénotypes distincts en ce qui concerne la couleur et la structure de l'écorce; chez les uns, l'écorce est claire et crevassée; chez les autres, elle est foncée et relativement régulière.

\begin{tabular}{|c|c|c|}
\hline Critères & Station 1 & Station 2 \\
\hline Localisation . . . . . . . . . . . . . & $\begin{array}{l}\text { Flanc nord du Djebel Bekker, } \\
\text { à proximité d'un ravin }\end{array}$ & $\begin{array}{l}\text { Oued el Anser, au pied du Djebel } \\
\text { Bezeze. Exposition générale nord } \\
\text { (variantes nord-est et est) }\end{array}$ \\
\hline Altitude moyenne $\ldots \ldots \ldots \ldots \ldots$ & $1590-1600 \mathrm{~m}$ & $1500 \mathrm{~m}$ \\
\hline Description $\ldots \ldots \ldots \ldots \ldots \ldots$ & $\begin{array}{l}\text { Cédraie mixte à Chêne vert } \\
\text { (sous-étage) } \\
\text { Futaie plus ou moins irrégulière, } \\
\text { belles taches de régénération } \\
\text { (fourré et gaulis) dans les trouées } \\
\text { d'abattage ; présence de semis } \\
\text { sous les arbres de futaie. Sujet } \\
\text { moyen: hauteur totale de } 20 \mathrm{~m} \text {, } \\
\text { diamètre de } 42 \mathrm{~cm} \text {. Carotte de } \\
\text { sondage : âge de } 200 \text { ans ; } \\
\text { alternance de périodes aux cernes } \\
\text { larges (o-20 ans, } 30-120 \text { ans) et } \\
\text { de périodes aux cernes fins } \\
\text { (20-30 ans, } 120-200 \text { ans). } \\
\text { Croissance radiale lente à partir } \\
\text { de } 120 \text { ans }\end{array}$ & $\begin{array}{l}\text { Cédraie mixte (Chêne vert, Frêne } \\
\text { dimorphe) où l'ambiance } \\
\text { est franchement mésophile. } \\
\text { Futaie plus ou moins irrégulière, } \\
\text { constituée de la juxtaposition } \\
\text { d'un étage dominant de futaie } \\
\text { âgée, aux arbres à la cime } \\
\text { tabulaire, et d'une régénération } \\
\text { de tous âges et de toutes } \\
\text { dimensions, notamment dans les } \\
\text { trouées. Étude de souche : âge } \\
\text { d'environ } 280 \text { ans (voir fig. } 4 \text {, } \\
\text { ci-contre, pour la croissance en } \\
\text { diamètre). } \\
\text { À retenir comme peuplement } \\
\text { semencier }\end{array}$ \\
\hline
\end{tabular}




\section{Station 3}

Versant sud du Djebel Chélia, tête d'un ravin à $300 \mathrm{~m}$ environ d'une parcelle de régénération ONTF, exposition nord-est

$1700 \mathrm{~m}$

Calcaire ou gréso-calcaire fissuré, présence d'éboulis

Cédraie pure ${ }^{(2)}$ (sous-étage de Chêne vert, pas de Pin d'Alep). Arbres de très belle venue au fût bien élagué. Présence de régénération de tous âges. Arbre moyen d'une hauteur totale de $30 \mathrm{~m}$, et d'un diamètre à hauteur d'homme de $55 \mathrm{~cm}$. Sur une un diamètre de $28 \mathrm{~cm}$ à hauteur d'abattage

Phénotype remarquable (vigueur et forme)

À retenir comme peuplement semencier souche de 60 ans, on a mesuré

Tableau lb Principales caractéristiques des stations du massif de Chélia

$1000 \mathrm{~mm}$ en fonction de l'altitude et de l'exposition ; c'est le domaine des étages bioclimatiques subhumide (principalement) et humide froids. Au plan géologique, on y rencontre principalement des substrats siliceux (grès et quartzites). Les peuplements sont, généralement, en bon état et de très beaux phénotypes (hauteur et forme) peuvent s'observer, notamment en altitude.

\section{Les Ouled Yacoub (Aurès oriental)}

Ce massif, de plus de 3000 ha, se situe dans la région de Khenchela, à une centaine de kilomètres à l'est de Batna. Le bioclimat subhumide y est le plus fréquent ; on y rencontre des substrats géologiques variés : grès calcaire, calcaire, poches de marnes localisées, schistes. La cédraie caractéristique dans ce massif se présente sous la forme de peuplements mixtes, le Cèdre en étage principal, le Chêne vert et le Frêne dimorphe en sous-étage. Des arbres remarquables (plus de $30 \mathrm{~m}$ de haut) se rencontrent souvent dans les bas-fonds et ravines où le sol est généralement plus profond.

\section{FIGURE 4}

CROISSANCE ET ACCROISSEMENT EN DIAMÈTRE D'UN ARBRE DE 280 ANS (Ouled Yacoub, station 2)

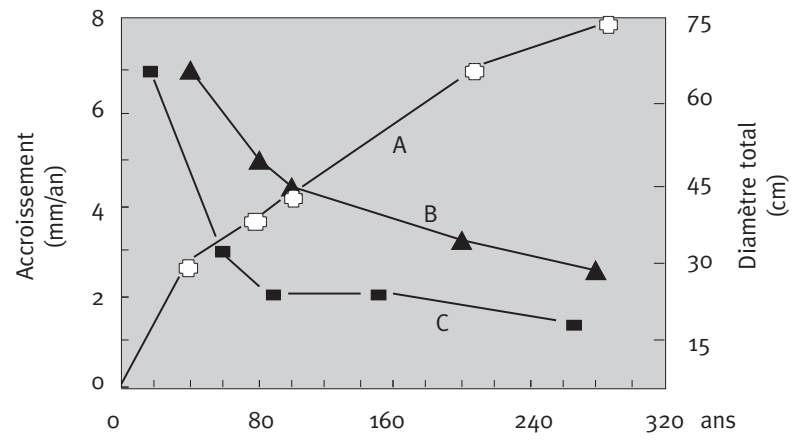

A : Croissance totale

B : Accroissement moyen annuel

$C$ : Accroissement courant annuel

(o-40 ans, 40-80 ans, 80-100 ans, 100-200 ans, 200-280 ans)

d'homme de $43 \mathrm{~cm}$

au phénotype (croissance

et forme) remarquable.

À retenir comme peuplement semencier
TABleau Ic

Principales caractéristiques des stations du massif de Ouled Yacoub 


\section{SYNTHÈSE DES PROSPECTIONS}

D’un point de vue sylvicole, la cédraie du Bélézma se caractérise par un déséquilibre notable des classes d'âge, les classes âgées ou vieillissantes étant largement en excès. L'absence presque totale de régénération naturelle et l'état de dégradation des sols dans une grande partie du massif posent le problème de cette cédraie de 7000 ha en termes de survie. Étant donné l'âge avancé de la plupart des arbres, les problèmes d'ordre sanitaire se posent de plus en plus avec acuité. En particulier, des infestations cryptogamiques (probablement dues à l'armillaire) sont périodiquement signalées et la mortalité dans les peuplements prend parfois des proportions inquiétantes. Les facteurs aggravants nous semblent être le pâturage et le taux de fréquentation du massif par le public, notamment depuis la réalisation d'aménagements récréatifs dans certaines parties du parc.

La cédraie à Chélia présente un aspect plus rassurant qu'au Bélézma. Le potentiel de renouvellement des stations semble conservé et l'espèce y manifeste une grande aptitude à coloniser l'espace dès que les conditions de sol et de lumière sont propices; les trouées d'abattage et les talus de piste, notamment, sont envahis par une régénération parfois dense. Comparativement, les peuplements sont en meilleur équilibre que dans le massif précédent.

Comme à Chélia, la cédraie dans les Ouled Yacoub présente globalement un état général satisfaisant. La régénération naturelle n'est pas rare ; elle est même souvent dense dans les trouées

\section{FIGURE 5}

\section{ORGANIGRAMME DU PROGRAMME DE CRÉATION D’UN VERGER À GRAINES DE FAMILLES POUR LA FORÊT DES OULED YACOUB}

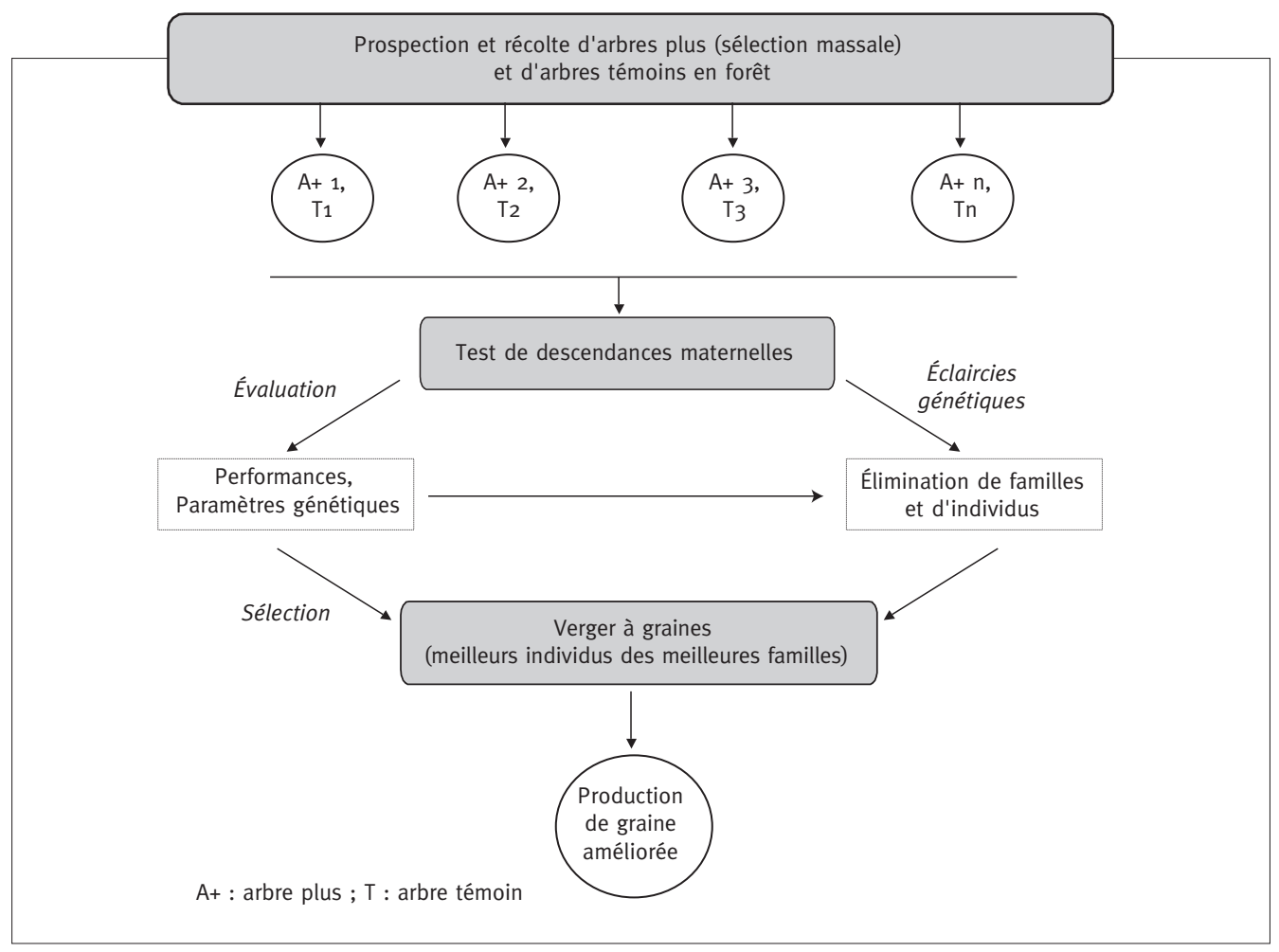


et, parfois, sous couvert de Chêne vert. Au plan sanitaire, aucune attaque sérieuse n'est actuellement à déplorer. Par contre, certaines parties du massif supportent une grande charge de bétail, ce qui ne va pas sans poser problème aussi bien pour les jeunes recrûs que pour les arbres adultes dont certains sont sévèrement ébranchés en période de disette, lorsque la ressource fourragère se fait rare au sol.

Les massifs prospectés recèlent des ressources génétiques très intéressantes qui méritent d'être conservées in situ et ex situ. Elles doivent être gérées spécialement pour produire de la semence à court terme (peuplements semenciers) aux fins d'approvisionnement des reboisements en cours et pour constituer un matériel de base pour le programme local d'amélioration de l'espèce. Certains peuplements du Djebel Bordjem dans le Bélézma, de l'oued Tidder dans le Chélia, des oueds El Anser et Zarif (Aïn Fakroun) dans les Ouled Yacoub présentent des phénotypes remarquables en ce qui concerne la vigueur et la forme. Ces trois massifs doivent, à cet égard, constituer l'épine dorsale du programme mais certaines populations marginales ou plus méridionales peuvent présenter des qualités recherchées en ce qui concerne des critères d'adaptation importants comme la résistance à la sécheresse, la croissance sur les marnes et calcaires ou la résistance à des insectes et maladies.

\section{VERS LA CRÉATION D’UN VERGER À GRAINES DE FAMILLES}

Dans les régions d'Algérie où il prospère, et plus encore dans l'Aurès en raison des étendues qu'il occupe, le Cèdre de l'Atlas est une composante écologique et socio-économique essentielle. La plupart des peuplements de cette espèce sont, à l'heure actuelle, gérés dans le cadre de parcs nationaux (Bélézma, Djurdjura, Ouarsenis, Atlas blidéen) où l'objectif principal est la recherche d'un équilibre entre conservation et développement. Des dérives protectionnistes constituent, dans certains cas, des contraintes à une gestion rationnelle de cette ressource génétique dont l'utilisation doit dépasser les cadres régional et national. Si les cédraies de l'Atlas tellien semblent toujours adaptées au climat contemporain, certains peuplements de l'Atlas saharien, notamment les plus méridionaux, se régénèrent difficilement ; les conditions qui ont vu leur installation ne sont, probablement, plus celles auxquelles ils sont soumis aujourd'hui, en raison d'une évolution du climat vers l'aridité.

Les mesures de conservation in situ des ressources génétiques du Cèdre dans le cadre des parcs nationaux ne peuvent, à elles seules, assurer la sauvegarde et la pérennité de l'espèce. D'abord, en raison des changements climatiques et de la régression des milieux naturels, ensuite à cause d'une pression humaine de plus en plus difficile à contenir. Aussi, la conservation et l'utilisation ex situ de ces ressources génétiques sur une large échelle aussi bien en Algérie que dans certains pays du pourtour méditerranéen, notamment la France, sont-elles urgentes et souhaitables.

La première phase du programme concerne la forêt des Ouled Yacoub, qui s'étend sur plus de 3000 ha. Il s'agira de constituer une population de production de graines susceptible d'approvisionner les reboisements en Cèdre à l'échelle du massif (figure 5, p. 120).

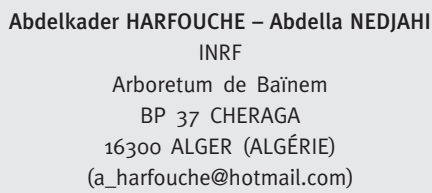




\section{BIBLIOGRAPHIE}

ABDESSEMED (K.). - Le Cèdre de l'Atlas (Cedrus atlantica Manetti) dans les massifs de l'Aurès et du Bélézma. Étude phytosociologique. Problèmes de conservation et d'aménagement. - Université d'AixMarseille, 1981. - 199 p. (Thèse de docteur-ingénieur).

ARBEZ (M.), FERRANDÈS (P.), UYAR (N.). - Contribution à l'étude de la variabilité géographique des Cèdres. - Annales des Sciences forestières, vol. 35, $\mathrm{n}^{\circ}$ 4, 1978, pp. 265-284.

BARITEAU (M.), FERRANDÈS (P.). - Les Cèdres. In : Amélioration des espèces végétales cultivées, chap. 8 : les espèces forestières / A. Gallais, H. Bannerot, Eds.. - 1992. - pp. 733-743.

BERKA (S.). - Étude de la variabilité intra-spécifique de Cedrus atlantica Manetti en Algérie par l'étude des stomates. - Annales de la Recherche forestière en Algérie, $\mathrm{n}^{\circ}$ 1, Nouvelle série, 1997, pp. 12-23.

DERRIDJ (A.). - Étude de la variabilité géographique des dimensions des pollens du Cèdre de l'Atlas (Cedrus atlantica Manetti) en Algérie. In : Symposium international sur le Cèdre, FAO/IUFRO. Antalya, Turquie, octobre 1990. - pp. 904-932.

FERRANDÈS (P.). - Cèdres. - Revue forestière française, vol. XXXVIII, nº spécial “Amélioration génétique des arbres forestiers", 1986, pp. 139-141.

HARFOUCHE (A.). - Étude comparative de quelques provenances algériennes de Cedrus atlantica Manetti. Étude des graines. - Annales de la Recherche forestière en Algérie, 1992, pp. 61-83.

MAIRE (R.). - Notice de la carte phytogéographique de l'Algérie et de la Tunisie. - Service cartographique de la Direction de l'Agriculture, du Commerce et de la Colonisation, Gouvernement général de l'Algérie, 1926.

NEDJAHI (A.). - La Cédraie de Chréa (Atlas blidéen) : Phénologie, Productivité, Régénération. - Université de Nancy I, 1988 (Thèse de Doctorat).

PRADAL (F.). - Variabilité génétique et écophysiologique du Cèdre. - Nogent-sur-Vernisson : ENITEF, 1979. - 87 p. (Mémoire).

TOTH (J.). - La Cédraie de la Verne-Ragusse dans le massif des Maures. - Revue forestière française, vol. XXV, $\mathrm{n}^{\circ}$ 2, 1973, pp. 115-120.

TOTH (J.). - Le Cèdre dans quelques pays du pourtour méditerranéen et dans deux autres pays à grande importance forestière. - Forêt méditerranéenne, tome II, $\mathrm{n}^{\circ} 1$, 1980, pp. 23-30.

ZOBEL (B.), TALBERT (J.). - Applied Forest Tree improvement. - New-York : John Wiley \& Sons, 1984. $504 \mathrm{p}$.

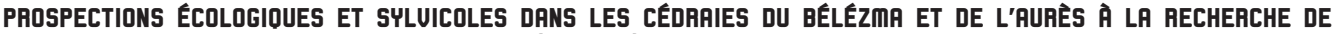 PEUPLEMENTS SEmENCIERS ET D'RRBRES PLUS [Résumé]}

Le Cèdre de l'Atlas est une essence forestière majeure en Algérie où elle s'étend sur près de 30 ooo ha. La configuration de l'aire naturelle de l'espèce et sa variabilité écologique ont favorisé une différenciation de morphotypes divers et d'écotypes adaptés à une gamme étendue de climats et de substrats géologiques. Ces caractéristiques constituent un atout pour la reconstitution des cédraies et le reboisement dans les Atlas tellien et saharien. Des prospections dans les massifs du Bélézma et de l'Aurès ont montré l'existence de peuplements et d'individus phénotypiquement remarquables pouvant servir de semenciers pour les projets de reboisement à court terme et de matériel de base pour le programme local d'amélioration de l'espèce. Afin de sauvegarder cette ressource génétique, dont l'intérêt dépasse le cadre national, des mesures de conservation et d'utilisation ex situ, aussi bien en Algérie que dans certains pays du pourtour méditerranéen, sont à envisager dans le cadre d’une coopération méditerranéenne.

PROSPECTINGS FOR ECOLOGICAL AND SILUICULTURAL PURPOSES IN THE CEDAR FORESTS OF BÉLÉmR AND RURÈS IN SERRCH OF SEED-PRODUCING STRNDS AND PLUS TREES [Abstract]

The Atlas cedar is a major forest species in Algeria. Its natural area is discontinuous and spreads over a wide range of ecological conditions resulting in differentiation of morphotypes and ecotypes adapted to various climates and geological substrata, which is valuable for use in reconstitution of native forests and afforestation of lands in the Tellian and Saharan Atlas mountains. Prospectings made in the Bélézma and Aurès cedar forests revealed good phenotypes which could be used in short term cedar plantation programs and as a base material for the local program of improvement for the species. In order to preserve this valuable and relatively endangered resource, measures have to be taken for its ex situ conservation and utilisation, as well as in Algeria or in other Mediterranean countries within the framework of joint networks. 